\title{
Roughness of Oblique Cutting of Sheet Metal Based on Dimensional Analysis
}

\author{
Jing Tao ${ }^{1}$, Zhengwei Fu ${ }^{1,2, *}$ and Boyuan Zhang ${ }^{3}$ \\ ${ }^{1}$ School of Mechanical and Electrical Engineering, Hubei Polytechnic University, Huangshi 435003, China \\ ${ }^{2}$ School of Machinery and Automation, Wuhan University of Science and Technology, Wuhan 430081, China \\ ${ }^{3}$ School of Electronic Engineering and Computer Science, Queen Mary University of London, London, E1 4FZ, United Kingdom
}

Received 3 August 2019; Accepted 7 November 2019

\begin{abstract}
The quality of the oblique cutting of sheet metal is difficult to improve by controlling the correlation factors that are numerous and coupled and the highly nonlinear factors that affect the roughness of the oblique cutting. To determine the influence of relevant factors on and obtain the mathematical model of the roughness of the oblique cutting, the influence order of each factor was first determined by significance analysis based on the orthogonal test. Subsequently, the dimensional relationship between the roughness of the oblique cutting and the related influencing factors was established. The dimensionless mathematical model between the shear plane roughness and the factors was derived using dimensional analysis. Using Datafit and orthogonal experimental data as basis, relevant parameters of the dimensionless mathematical model were also determined by fitting. The quantitative relationship between the roughness of the oblique cutting and relevant physical quantities was then obtained. Finally, the mathematical model of the roughness of the oblique cutting was verified in the field. Results indicate that the influence of relevant factors on the roughness of the oblique cutting follows the order of sheet thickness $>$ cutter head clearance $>$ material $>$ shear angle. Moreover, the mathematical model of the roughness of the oblique cutting established by orthogonal test and dimensional analysis can accurately reflect the internal relationship of the factors affecting the shear plane. The relative error of the roughness model of the oblique cutting is only 0.7 . The study provides a theoretical basis for improving the quality of oblique cutting and design of oblique edge shearer.
\end{abstract}

Keywords: Oblique cutting, Dimensional analysis, Orthogonal test

\section{Introduction}

With characteristics of low shearing force, light weight, and low energy consumption [1], oblique cutting shearers are widely used worldwide. However, selecting parameters during oblique cutting for sheet metal is difficult. This difficulty often results in burr, folded edge, contact mark, and even shear plane failure [2] and leads to failure in meeting the precision requirement. This failure occurs because the shear plane roughness after the sheet metal is sheared by the existing shearer. To address this issue, a growing number of scholars aim at developing a shearer with increased precision, commonly using the single variable method. This method improves the accuracy of the oblique cutting by controlling a single influencing factor, such as shear clearance or shear angle. However, the actual effect of controlling shear quality using a single influencing factor is unsatisfactory.

Therefore, understanding the bending forming law and improving the bending forming accuracy are urgent concerns in the bending industry. Therefore, scholars have started to seek the rule of defect formation in sheet metal shearing through theoretical and finite element analyses [34]. Still, the quality of the shear plane is difficult to improve by controlling the correlation factors that are numerous and

*E-mail address: fu1065171787@163.com

ISSN: $1791-2377 @ 2019$ School of Science, IHU. All rights reserved.

doi:10.25103/iestr.125.22 coupled and the highly nonlinear factors that affect the roughness of the shear plane. Hence, the establishment of the joint adjustment of multi factors to shear quality is the common development trend of the current shear industry.

For this purpose, the importance of each influencing factor to shear plane roughness is determined through orthogonal test and significance analysis. Subsequently, dimensional analysis is performed and combined with Datafit for data fitting, thereby establishing the mathematical model of oblique cutting roughness. The aim is to establish the quantitative relationship between the roughness of the oblique cutting and the influencing factors to provide theoretical support for improving the shear quality of the oblique cutting and obtain a high shear quality by determining each physical quantity through the model.

\section{State of the art}

To date, many scholars have conducted numerous studies on improving shear quality. Sergei Senn et al. [5] optimized the tool geometry to improve the shear quality through Latin hypercube variation sampling. This model optimizes the shear edge but does not consider the joint influence of multiple factors on the shear plane roughness. Paetzold I et al. [6] performed the fatigue test on sheets and obtained the optimal values of various factors affecting the shear performance through the evaluation of the experimental results. The scheme is expensive, lengthy, and ineffective 
though. Cheon S et al. [7] predicted the tool life during sheet shearing by optimizing the tool wear model. The replacement time of the tool was proposed, but the shear quality was not substantially improved when the tool was intact. Didin Zakariya Lubis et al. [8] studied the influence of shear clearance and velocity on the shear quality of copper sheets and determined the optimal shear clearance and velocity. They only focused on a single copper sheet, so the result is not universal. Soares J A et al. [9] determined the influence rule of shear clearance on shear quality through field tests and ANSYS numerical simulation. The accuracy of shear plane is clearly difficult to improve through a single influencing factor. Husson $\mathrm{C}$ et al. [10] established the influence of clearance and prop wear on blanking quality through simulation and experiment. However, they did not provide a specific mathematical model of relevant influence factors, which was of no practical significance to the improvement of shear quality. $\mathrm{H}$ Arfa et al. [11] established the optimal cutting path to improve the metal shear quality through the finite element modeling and simulation of the sheet shear molding process. They only conducted a theoretical study and ignored the actual situation. Sutasn Thipprakmas et al. [12] optimized burr scraping through finite element software, but the scheme could not optimize and improve the shear plane accuracy from the source. Wu Shengchao et al. [13] used numerical simulation and experiment to determine the optimal process parameters of shear plane roughness, but this method was carried out under specific conditions that were not representative. Kang Feng et al. [14] used finite element simulation to determine the core influencing factors of sheet shear quality but did not provide a quantitative mathematical model for improving shear accuracy. Yue Xuehu et al. [15] established the influence of the blank holder force on shear quality through the simulation model but encountered difficulty in the conduction and control of the internal force of the sheet metal. Han Jiang et al. [16] determined the influence rules of various factors on shear quality through the DEFORM-3D simulation experiment and the parameters of the optimal shear process by using a quadratic programming function. The study on the influencing factors of shear plane through this method is not comprehensive, so the actual effect is not ideal. Nie Jingliang et al. [17] used finite element analysis software to simulate and optimize the existing shear characteristics of low efficiency and high speed to make the shear stress distribution even and improve the quality of the shear plane. However, this method does not significantly improve the accuracy of the shear plane in the actual production process. Li Zhongrong et al. [18] studied the influence of tools with different plane roughness values on shear quality and verified the inverse relationship between tool roughness and shear quality through field tests. Nevertheless, the tool plane roughness is difficult to control accurately, making the shear quality difficult to control as well. Shi Yu et al. [19] used the ANSYS software to simulate the sheet shear molding process and determined the distribution rule of the stress-strain of materials in the shear process to improve the shear quality by optimizing the shear force, but the distribution of the force in the shear process is difficult to control.

The above studies on shear plane roughness mainly simulate the shearing of sheet metal through a simulation software and improve the shear quality by optimizing a single influencing factor through tests. Few studies focused on the interaction between shear plane roughness and multiple physical quantities, and few quantitative models can identify the relationship between shear plane roughness and various factors. To address the shortcomings of the above studies, the order of influence of various related factors on the roughness of the oblique cutting is determined by orthogonal test and dimensional analysis, and the quantitative model of roughness is established, providing a theoretical basis for improving the shear quality of the oblique cutting.

The remainder of this study is organized as follows. In Section 3, the influence sequence of various factors on the roughness of the oblique cutting is determined through orthogonal test and significance analysis, the quantitative model of the roughness of the oblique cutting is established by dimensional analysis and Datafit. In Section 4, the quantitative model of roughness of the oblique cutting is verified by data fitting and field test. In the last section, the study is summarized and conclusions are drawn.

\section{Methodology}

\subsection{Significance verification of orthogonal test}

The orthogonal test can obtain the optimal collocation scheme through few experiments and can thus reflect the internal relationship between the roughness of the shear plane of the oblique cutting and each influencing factor. To facilitate the significance verification, a column of error terms is added. To determine the importance of the influence of each factor on the roughness of the oblique cutting, variance analysis and significance verification should be performed on the orthogonal test data. The influence of each factor on the results should be determined by analyzing the contribution of the difference change caused by each factor to the total change.

The sum of the squares of deviations caused by each factor is shown in Equation (1).

$$
S S_{j}=\frac{r}{n} \sum_{i=1}^{r}(k-\bar{y})^{2}=\frac{r}{n}\left(\sum_{i=1}^{r} K^{2}\right)-\frac{1}{n}\left(\sum_{i=1}^{n} y_{i}\right)^{2}
$$

where $S S_{j}$ influences the sum of the squares of deviations of factor $j, n$ is the total number of orthogonal tests, $i$ is the number of tests, $y_{i}$ is the value of the $i$ test, $\bar{y}=\frac{1}{n} \sum_{i=1}^{n} y_{i}$ is the mean value of the tests, $K$ represents the sum of the experimental data of the same level in the same column, $k$ is the mean value of the experimental data of the same level in the same column, and $r$ is the level number of orthogonal tests.

To reduce the influence of the level number of the orthogonal test on the sum of the squares of deviations, the degree of freedom is introduced to calculate the mean square. The degree of freedom of any factor is expressed as Equation (2).

$d f_{j}=r-1$

Then, the mean square of the factor is shown in Equation (3).

$$
M S_{j}=\frac{S S_{j}}{d f_{j}}
$$


where $M S_{j}$ is the mean square of influence factor $j$.

According to the mean square, the $F$ value is calculated using Equation (4):

$F_{j}=\frac{M S_{j}}{M S_{e}}=\frac{S S_{j} / d f_{j}}{S S_{e} / d f_{e}}$

where $e$ is the error term.

\subsection{Establishment of dimensional model}

In the previous section, the influence of each factor on the roughness of the oblique cutting is clarified. To establish the quantitative relationship between the roughness of the sheet metal after being sheared by oblique cutting and various factors, this study introduces dimensional analysis to model the shear plane roughness; dimensional analysis can express the intercoupling of multiple physical influencing factors in a simple mathematical model and reduce the number of variables [20]. In the model, the five variables of thickness $(h)$, tool edge clearance $(d)$, shear velocity $(v)$, the sheet material properties and shear angle $(\theta)$ are selected to establish a quantitative relationship with shear plane roughness $(R a)$, and the material properties are expressed as tensile strength limit $\left(\sigma_{b}\right)$, elastic modulus $(E)$, and elongation $(\delta)$. The functional relation of the roughness of the oblique cutting is established according to the physical quantity, as shown in Equation (5).

$$
R a=\varphi\left(h, d, v, \theta, \sigma_{b}, E, \delta\right)
$$

Equation (5) finds the relation of shear plane roughness and is transformed into a general form, as shown in Equation (6).

$$
f\left(h, d, v, \theta, \sigma_{b}, E, \delta, R a\right)=0
$$

where $f$ is an unknown function.

Eight physical quantities in Equation (6) are modeled according to the Buckingham Pi theorem. In Equation (6), shear angle $\theta$ and elongation $\delta$ are removed as dimensionless quantities, and the remaining six physical quantities are expressed in dimensional form with length $L$, time $T$, and mass $M$ as the basic dimensions, as shown in Table 1 [21].

Table 1. Dimension correspondence table

\begin{tabular}{c|c|c|c|c|c|c}
\hline $\begin{array}{c}\text { Physical } \\
\text { Quantity }\end{array}$ & $h$ & $d$ & $v$ & $\sigma_{b}$ & $E$ & $R a$ \\
\hline Dimension & $L$ & $L$ & $L T^{-1}$ & $M L^{-1} T^{-2}$ & $M L^{-1} T^{-2}$ & $M L T^{-2}$ \\
\hline
\end{tabular}

The corresponding dimensional matrix $\left(A_{3 \times 6}\right)$ in Table 1 is shown in Equation (7).

$$
\begin{aligned}
& (h)(d)(v)\left(\sigma_{b}\right)(E)(R a) \\
& A_{3 \times 6}=\left|\begin{array}{cccccc|c}
0 & 0 & 0 & 1 & 1 & 0 & M \\
1 & 1 & 1 & -1 & -1 & 1 \\
0 & 0 & -1 & -2 & -2 & 0
\end{array}\right| T
\end{aligned}
$$

The homogeneous equation is formed by the matrix ( $\left.Y=\left(y_{1}, y_{2}, y_{3}, y_{4}, y_{5}, y_{6}\right)^{\mathrm{T}}\right)$ constructed according to the dimensional homogeneous principle and by matrix $A_{3 \times 6}$, as shown in Equation (8).
$A Y=0$

Given that the rank of matrix $A_{3 \times 6}$ is 3 , Equation (8) has three basic solutions, which are expressed in Equation (9).

$Y_{1}=\left[\begin{array}{c}-1 \\ 0 \\ 0 \\ 0 \\ 0 \\ 1\end{array}\right] \quad Y_{2}=\left[\begin{array}{c}-1 \\ 1 \\ 0 \\ 0 \\ 0 \\ 0\end{array}\right] \quad Y_{3}=\left[\begin{array}{c}0 \\ 0 \\ 0 \\ 1 \\ -1 \\ 0\end{array}\right]$

The three independent dimensionless quantities established by Equation (9) are shown in Equation (10).

$\left\{\begin{array}{l}\pi_{1}=h^{-1} d^{0} v^{0} \sigma_{b}^{0} E^{0} R a^{1}=\frac{R a}{h} \\ \pi_{2}=h^{-1} d^{1} v^{0} \sigma_{b}^{0} E^{0} R a^{0}=\frac{d}{h} \\ \pi_{3}=h^{0} d^{0} v^{0} \sigma_{b}^{1} E^{-1} R a^{0}=\frac{\sigma_{b}}{E}\end{array}\right.$

Shear angle $\theta$ and material elongation $\delta$ are also independent dimensionless, as shown in Equation (11).

$\left\{\begin{array}{l}\pi_{4}=\theta \\ \pi_{5}=\delta\end{array}\right.$

According to the Buckingham Pi theorem, an unknown function Equation (12) that is equivalent to Equation (6) exists.

$F\left(\pi_{1}, \pi_{2}, \pi_{3}, \pi_{4}, \pi_{5}\right)=0$

Equation (12) is transformed into Equation (13) and substituted into Equations (10) and (11).

$\pi_{1}=\psi\left(\pi_{2}, \pi_{3}, \pi_{4}, \pi_{5}\right) \Rightarrow \frac{R a}{h}=\psi\left(\frac{d}{h}, \frac{\sigma_{b}}{E}, \theta, \delta\right)$

where $\psi$ is a dimensionless function of the oblique cutting roughness and an unknown quantity.

The roughness of the oblique cutting can be expressed by Equation (14).

$R a=h \psi\left(\frac{d}{h}, \frac{\sigma_{b}}{E}, \theta, \delta\right)$

\subsection{Orthogonal experimental design}

Many factors influence the roughness of the shear plane when the sheet metal is sheared by oblique cutting, and the degrees of influence vary. In this study, according to field practice and working experience, five main influencing factors are established: sheet thickness $(h)$, tool edge clearance $(d)$, shear velocity $(v)$, shear angle $(\theta)$, and material properties of the sheet. Minor influencing factors, such as sheet length and material anisotropy are ignored.

To establish the influence degree of the five factors on the roughness of the oblique cutting, an orthogonal test of the five factors and five levels is conducted in the HGS62/13 Numerical Control Sheet Shear Center. In 
addition, to facilitate the significance verification, a column of data is added for the error item to form an orthogonal table $L_{25}\left(5^{6}\right)$ to record the experimental data. After shear completion, the roughness $(R a)$ of the shear plane is measured by the roughness measuring instrument. The specific test data are shown in Table 2.

Table 2. Design table of orthogonal test of oblique cutting

\begin{tabular}{|c|c|c|c|c|c|c|c|}
\hline Test No. & $\begin{array}{l}\text { Tool Edge } \\
\text { Clearance } \\
(\mathrm{mm})\end{array}$ & $\begin{array}{l}\text { Sheet } \\
\text { Thickness } \\
(\mathrm{mm})\end{array}$ & $\begin{array}{l}\text { Shear Angle } \\
\left({ }^{\circ}\right)\end{array}$ & Material & $\begin{array}{l}\text { Shear Velocity } \\
(\mathrm{m} / \mathrm{s})\end{array}$ & Error Term & Roughness \\
\hline 1 & 0.5 & 6 & 0.5 & Q195A & 3 & 1 & 26.8 \\
\hline 2 & 0.5 & 7 & 1 & Q345B & 3.5 & 2 & 36.3 \\
\hline 3 & 0.5 & 8 & 1.5 & 45 & 4 & 3 & 34.5 \\
\hline 4 & 0.5 & 9 & 2 & 60 & 4.5 & 4 & 23.4 \\
\hline 5 & 0.5 & 10 & 2.5 & Q235A & 5 & 5 & 34.6 \\
\hline 6 & 0.6 & 6 & 1 & 45 & 4.5 & 5 & 26.8 \\
\hline 7 & 0.6 & 7 & 1.5 & 60 & 5 & 1 & 28.2 \\
\hline 8 & 0.6 & 8 & 2 & Q235A & 3 & 2 & 22.3 \\
\hline 9 & 0.6 & 9 & 2.5 & Q195A & 3.5 & 3 & 20.9 \\
\hline 10 & 0.6 & 10 & 0.5 & Q345B & 4 & 4 & 32.8 \\
\hline 11 & 0.7 & 6 & 1.5 & Q235A & 3.5 & 4 & 29.2 \\
\hline 12 & 0.7 & 7 & 2 & Q195A & 4 & 5 & 23.6 \\
\hline 13 & 0.7 & 8 & 2.5 & Q345B & 4.5 & 1 & 34.5 \\
\hline 14 & 0.7 & 9 & 0.5 & 45 & 5 & 2 & 26.4 \\
\hline 15 & 0.7 & 10 & 1 & 60 & 3 & 3 & 35.5 \\
\hline 16 & 0.8 & 6 & 2 & Q345B & 5 & 3 & 27.6 \\
\hline 17 & 0.8 & 7 & 2.5 & 45 & 3 & 4 & 28.5 \\
\hline 18 & 0.8 & 8 & 0.5 & 60 & 3.5 & 5 & 23.8 \\
\hline 19 & 0.8 & 9 & 1 & Q235A & 4 & 1 & 26.7 \\
\hline 20 & 0.8 & 10 & 1.5 & Q195A & 4.5 & 2 & 28.9 \\
\hline 21 & 0.9 & 6 & 2.5 & 60 & 4 & 2 & 24.1 \\
\hline 22 & 0.9 & 7 & 0.5 & Q235A & 4.5 & 3 & 26.3 \\
\hline 23 & 0.9 & 8 & 1 & Q195A & 5 & 4 & 23.3 \\
\hline 24 & 0.9 & 9 & 1.5 & Q345B & 3 & 5 & 22.8 \\
\hline 25 & 0.9 & 10 & 2 & 45 & 3.5 & 1 & 27.4 \\
\hline
\end{tabular}

3.4 Function fitting in the dimension model for roughness of oblique cutting

Equation (14) indicates that the roughness model of the oblique cutting is not only related to the physical quantity of the influencing factors but also has a great relationship with dimensionless coefficient function $\psi$. To determine the unknown dimensionless coefficient function $(\psi)$ of the roughness of the oblique cutting, the data collected from Table 2 of the orthogonal test are imported into Datafit for fitting, and the specific function form of the unknown dimensionless coefficient is obtained to determine the mathematical model of the roughness of the oblique cutting. The material properties selected in the orthogonal test are shown in Table 3.

Table 3. Properties of experimental shear materials

\begin{tabular}{l|l|l|l|l}
\hline Material Name & Tensile Strength (Pa) & Elasticity Modulus (Pa) & Elongation(\%) & $\begin{array}{l}\text { Ratio of Tensile Strength } \\
\text { to Elasticity Modulus }\end{array}$ \\
\hline Q195A & 430000000 & 200000000000 & 0.33 & 0.00215 \\
Q345B & 620000000 & 208000000000 & 0.21 & 0.002980769 \\
45 & 600000000 & 210000000000 & 0.16 & 0.002857143 \\
60 & 675000000 & 205000000000 & 0.12 & 0.003292683 \\
Q235A & 500000000 & 206000000000 & 0.26 & 0.002427184 \\
\hline
\end{tabular}

After the data in Table 2 are converted into the five dimensionless values (i.e., $\frac{R a}{h}, \frac{d}{h}, \frac{\sigma_{b}}{E}, \delta$, and $\theta$ ) in Equation (14), the data are imported into Datafit, and $\theta$ is expressed as the tangent value $(\psi)$ for convenience. Given that the dimensionless values of the four dependent variables are all less than 1, higher order terms can be ignored and general Equation (15) is selected for nonlinear fitting regression to determine function $\psi$.

$$
\begin{aligned}
y= & a_{1}+a_{2} x_{1}+a_{3} x_{1}^{2}+a_{4} x_{1}^{3}+b_{1} x_{2}^{1}+b_{2} x_{2}^{2}+b_{3} x_{2}^{3} \\
& +c_{1} x_{3}^{1}+c_{2} x_{3}^{2}+c_{3} x_{3}^{3}+d
\end{aligned}
$$

where a, b, c, d are the correlation coefficient, $x_{1}=\frac{d}{h}, x_{2}=\delta$, and $x_{3}=\tan \theta$.

The dimensional nonlinear mathematical model corresponding to Equation (6) is obtained, as shown in Equation (16).

$$
R a=h \times\left[\begin{array}{l}
-382.2\left(\frac{d}{h}\right)^{3}+297.2\left(\frac{d}{h}\right)^{2}-36.4\left(\frac{d}{h}\right) \\
+171692.1(\tan \theta)^{3}-14135.1(\tan \theta)^{2} \\
+334.5 \tan \theta-372307907.4\left(\frac{\sigma_{b}}{E}\right)^{3} \\
+1170399\left(\frac{\sigma_{b}}{E}\right)^{2}+2966.2\left(\frac{\sigma_{b}}{E}\right) \\
+186.2 \delta^{3}-121.7 \delta^{2}+29.2 \delta-8.9
\end{array}\right]
$$

\section{Result Analysis and Discussion}

4.1 Significance analysis for roughness of oblique cutting According to Equations (1) to (4), the corresponding values are calculated, and the critical value $\left(F_{\alpha}\left(d f_{j}, d f_{e}\right)\right)$ is consulted for significance analysis. When $F>F_{0.01}$, the factor has a highly significant influence on the result. When 
$F_{0.01}>F>F_{0.05}$, the factor has a significant influence on the result. When $F_{0.05}>F>F_{0.1}$, the factor has a certain influence on the result. When $F_{0.1}>F$, the factor has no significant influence on the result (See Table 4).

Table 4. Significance analysis of roughness of oblique cutting

\begin{tabular}{l|l|l|l|l|l|l}
\hline & $S S_{j}$ & $d f_{j}$ & $M S_{j}$ & $F_{j}$ & $F_{\alpha}\left(d f_{j}, d f_{e}\right)$ \\
\hline Tool edge & 136.7704 & 4 & 34.1926 & 6.01 & $F_{0.1}(4,4)=4.11$ \\
clearance/d & & & & & $F_{0.05}(4,4)=6.39$ \\
Sheet thickness $/ h$ & 422.3064 & 4 & 105.5766 & 18.56 & Influencing \\
Shear angle $/ \theta$ & 70.1544 & 4 & 17.5386 & 3.08 & $F_{0.01}(4,4)=15.98$ \\
Materials & 100.4824 & 4 & 25.1206 & 4.42 & & Highly significant \\
Shear velocity $/ v$ & 4.1744 & 4 & 1.0436 & 0.18 & Not significant \\
Error item $/ e$ & 22.7584 & 4 & 5.6896 & & Influencing \\
\hline
\end{tabular}

Table 4 indicates that among the five factors that affect the roughness of the oblique cutting, only sheet thickness $(h)$ has a highly significant influence. The greater the number of statistics $F_{j}$ is, the greater the influence is. Then, the order of influencing factors on the oblique cutting is sheet thickness $>$ cutter head clearance $>$ material $>$ shear angle $>$ shear velocity. In addition, in Table 4 , the mean square of shear velocity is less than the mean square of the error term, indicating that shear velocity has little influence on the roughness of the oblique cutting, which is a minor factor and can be ignored.

\subsection{Analysis on dimension model of roughness of oblique cutting}

Equation (14) shows that the roughness of the oblique cutting is associated with four factors, namely, cutter head clearance, thickness of the material, shear angle, and material properties, and the shear velocity set is not included in the dimensional analysis, indicating that the roughness of the shear plane has no relevance to the shear velocity when the sheet metal is sheared by oblique cutting, which is consistent with the result of verification $\mathrm{F}$ in the orthogonal test.

\subsection{Model fitting}

To observe the fitting situation of Equation (16) visually, the roughness in the orthogonal test (Table 1) is fitted with Equation (16), and the fitting control model is represented by Figure 1.

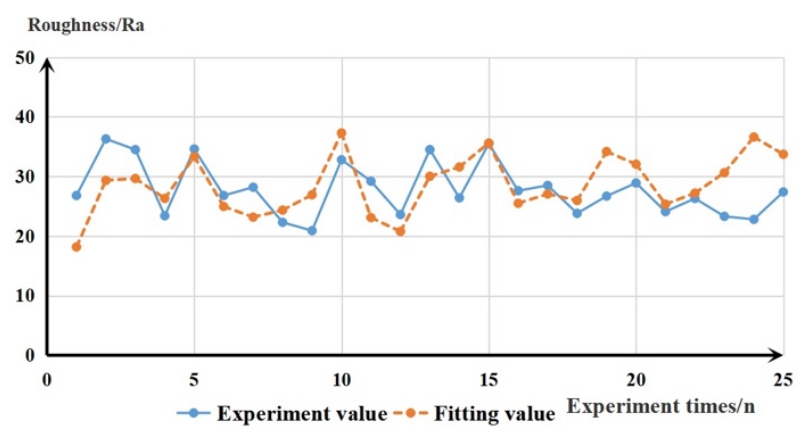

Fig 1. Fitting degree of roughness model and orthogonal experimental value

Figure 1 shows the roughness model of the oblique cutting obtained based on dimensional analysis after being fit by the Datafit software. The relative average error $(e=0.7)$ between the fitting model and the experimental data indicates that the roughness model has a good fitting effect with the actual roughness.

\subsection{Field verification}

To verify the correctness of the roughness model (Equation (16)), field test should be performed for field verification. Hgs62/13 CNC Numerical Control Sheet Shear Center is employed for field verification, as shown in Figure 2(a). For shearing, the following configuration is set: $16 \mathrm{MnNb}$ sheet thickness $h=8.5 \mathrm{~mm}$, cutter head clearance $d=0.65 \mathrm{~mm}$, shear angle $\theta=1.75^{\circ}$. The shear plane is shown in Figure 2(b).
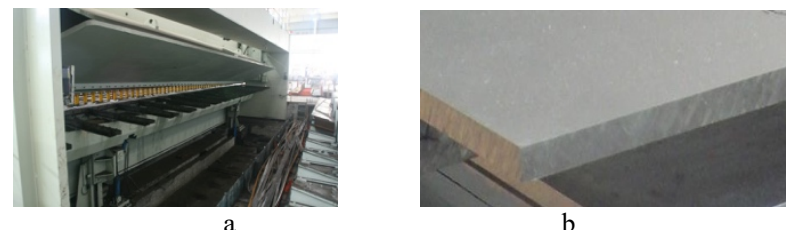

Fig 2. Shear field (a) and shear plane (b)

Shear velocity is taken as a single variable, and the field experiment is performed with a single variable. The fluctuation of the shear plane roughness of the sheet after being sheared is within $2 \%$, indicating that the shear velocity is independent of the shear plane roughness. In addition, the shear plane roughness of the sheet after being sheared by oblique cutting is $R a=31.8$, which is calculated as 30.7 by the roughness model (Equation (16)), and the relative error is $3.5 \%$, which conforms to the requirements of actual production accuracy.

\section{Conclusions}

To establish the relationship between the shear plane roughness of the oblique cutting and the influencing factors through orthogonal test and dimensional analysis, this study established the dimensionless mathematical model between the shear plane roughness and the influencing factors, and established the quantitative relationship between the shear plane roughness and the physical quantity using the Datafit software. Finally, the accuracy and practicability of the model are demonstrated through model fitting control and field experiment. After the above discussion, the following conclusions are drawn:

(1) Using orthogonal test and significance analysis, the influencing factors of the shear plane roughness of the oblique cutting follows the order sheet thickness $>$ cutter head clearance $>$ material $>$ shear angle.

(2) According to the orthogonal test and dimensional analysis, shear plane roughness is independent of shear velocity.

(3) Using dimensional analysis and Datafit, the calculation model of the roughness of the oblique cutting is derived, and the model can derive the roughness of the shear 
plane before shear and avoid being scrapped due to insufficient shear quality.

In this study, a quantitative model of the shear plane roughness of the oblique cutting is obtained by combining experiment and theory, which is of guiding significance to improving the setting of the relevant parameters of the shear plane roughness of the oblique cutting. However, this method is only applicable to a single type of oblique cutting shearer, and the shear plane roughness model of other types of oblique edge shearer must be re-established. Therefore, in future studies, the shear plane roughness model of each type must be established for the model to be directly invoked in the actual production process.

This is an Open Access article distributed under the terms of the Creative Commons Attribution License

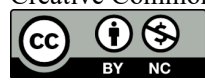

\section{Reference}

[1] Wu Zhengquan, Cheng Pengfei, Liu Jingxiang, et al, "Design of High Precision Consecutive Punching and Shearing Hydraulic System Based on PLC". Machine Tool \& Hydraulics, 47(10), 2017, pp.35-37.

[2] Zhang Liang, "Simulation and prediction of cross-section quality for pre-cut blanking based on BP neural network". Forging \& Stamping Technology, 43(12), 2018, pp.175-179.

[3] Ma Bing, Zhuang Feng, Chao Zhigang, et al, "Finite element analysis of shearing process of roll-cutting shear". China Metalforming Equipment \& Manufacturing Technology, 48(5), 2013, pp.66-68.

[4] Li Longrui, Zhang Xianglin, "Finite Element Simulation of Fine Blanking and Die Clearance". Journal of Netshape Forming Engineering, 03(1), 2011, pp.10-13.

[5] Sergei Senn, Mathias Liewald, "Numerical investigation of a new sheet metal shear cutting tool design to increase the part quality by superposed compression stress". Journal of Physics: Conference Series, 1063(1), 2018.

[6] Paetzold I, Dittmann F, Feistle M, et al, "Influence of shear cutting parameters on the fatigue behavior of a dual-phase steel". Journal of Physics: Conference Series, 896(1), 2017.

[7] Cheon S, Kim N, "Prediction of tool wear in the blanking process using updated geometry". Wear, 352, 2016, pp.160-170.

[8] Didin Zakariya Lubis, Muslim Mahardika, "Influence of Clearance and Punch Velocity on the Quality of Pure Thin Copper Sheets Blanked Parts". IOP Conference Series: Materials Science and Engineering, 157(1), 2016.

[9] Soares J A, Gipiela M L, Lajarin S F, et al, "Study of the punch die clearance influence on the sheared edge quality of thick sheets". The International Journal of Advanced Manufacturing Technology, 65(1-4), 2013, pp.451-457.

[10]Husson C, Correia J P M, Daridon L, et al, "Finite elements simulations of thin copper sheets blanking: Study of blanking parameters on sheared edge quality". Journal of materials processing technology, 199(1-3), 2008, pp.74-83.

[11]Arfa H, Bahloul R, BelHadjSalah H, "Finite element modelling and experimental investigation of single point incremental forming process of aluminum sheets: influence of process parameters on punch force monitoring and on mechanical and geometrical quality of parts". International journal of material forming, 6(4), 2013, pp.483-510.
[12] Sutasn Thipprakmas, Wiriyakorn Phanitwong, Mutjarin Chinwithee, et al, "Reciprocating Shaving Approach to Eliminate Crack and Burr Formations in Pressed Parts". Key Engineering Materials, 934, 2010, pp.201-206.

[13] Wu Shengchao, Wang Tao, Liu Hua, et al, "Simulation and Parameter Optimization of Blanking Process for Thick Plate". Journal of Netshape Forming Engineering, 10(1), 2018, pp.156160.

[14]Kang Feng, Zhou Jie, Quan Guozheng, et al, "Finite Element Simulation for Blanking Process of Thick Metal Plate and Parameter Optimization". China Metalforming Equipment \& Manufacturing Technology, 40(1), 2005, pp.66-68.

[15] Yue Xuehu, Fan Yujin, Zheng Huaihe, et al, "Influence of blank holder force on forming quality during blanking". Forging \& Stamping Technology, 43(4), 2018, pp.53-59.

[16] Han Jiang, Hu Ziyu, Zhang Kuibang, et al, "A prediction model study and optimization of shear section quality of sheet metal based on simulation". Journal of Hefei University of Technology (Natural Science), 41(12), 2018, pp.1585-1589.

[17] Nie Jingliang, Li Yugui, Xu Yurui, "Numerical simulated study on fracture of metal plate high-speed shearing". Heavy Machinery, (4), 2015, pp.48-52.

[18]Li Zhongrong, Zhang Longqiang, Yan Qiusheng, et al, "Effect of Tool Surface Roughness on Shear Quality of Electrical Steel". Mechanical \& Electrical Engineering Technology, 47(8), 2018, pp.11-15.

[19] Shi Yu, Meng Binbin, "Finite Element Simulation on Shearing Process for Steel Gratings Shearing Machine". Machine Tool \& Hydraulics, (2), 2015, pp.33-37.

[20]Fu Zemin, Mo Jianhua, Chen Wei, et al, "Springback Model for Air-bending of Sheet Metal Based on Dimensional Analysis Method". Journal of Mechanical Engineering, 46(12), 2010, pp.5358.

[21]Liang Canbin, Cao Zhoujian, Chen Zhitao, "Introduction to dimensional analysis". College Physics, 37(02), 2018, pp.12-17. 